TTK-12-02

\title{
Leptogenesis from Additional Higgs Doublets
}

\author{
Björn Garbrecht \\ Institut für Theoretische Teilchenphysik und Kosmologie, \\ RWTH Aachen University, 52056 Aachen, Germany
}

\begin{abstract}
Leptogenesis may be induced by the mixing of extra Higgs doublets with experimentally accessible masses. This mechanism relies on diagrammatic cuts that are kinematically forbidden in the vacuum but contribute at finite temperature. A resonant enhancement of the asymmetry occurs generically provided the dimensionless Yukawa and self-interactions are suppressed compared to those of the Standard Model Higgs field. This is in contrast to typical scenarios of Resonant Leptogenesis, where the asymmetry is enhanced by imposing a degeneracy of singlet neutrino masses.
\end{abstract}

\section{Introduction}

Experimentally accessible $C P$-violating effects, such as the mixing of neutral mesons, are often resonantly enhanced. This suggests that the matter-antimatter asymmetry of the Universe may have emerged from resonant mixing as well. The enhancement is maximized when the mixing states are nearly degenerate in their masses and couplings. For example, Resonant Leptogenesis [1 [5] relies on almost mass-degenerate singlet neutrinos $N$, where the masses are of Majorana type and violate lepton number. The degeneracy may result from an approximate global symmetry or from parametric tuning. One reason for introducing it is to allow for low reheat temperatures. Barring resonant enhancement, the observed asymmetry can only arise at temperatures above $10^{9} \mathrm{GeV}$, which lead to the overproduction of unwanted thermal relics in some Particle Physics scenarios.

In the most generic realizations, the couplings of the singlet neutrinos are so small that they escape detection in colliders, even if their masses are of Electroweak scale or within an experimentally accessible range above. (See, however, Ref. [6].) It is therefore interesting to consider the question whether particles that are not gauge singlets can lead to a resonantly enhanced asymmetry as well. Such particles could be found in collider experiments.

Obvious candidates are additional Higgs doublets $\phi$. These fields combine to gauge singlets with the Standard Model (SM) lepton doublets $\ell$, such that they can couple to 
a singlet neutrino at the renormalizable level. Due to its arbitrarily small couplings, the singlet may deviate from equilibrium in the Early Universe, thus breaking $T$ invariance, which is necessary in order to make the $C P$-violation needed for Leptogenesis effective, what would be barred by the CPT-theorem otherwise. The masses and couplings of the Higgs doublets do not violate lepton number, but generically violate lepton flavor. This implies that before washout, no lepton asymmetry, but only a flavor asymmetry can be generated. The flavor asymmetries suffer different amounts of washout, such that eventually, a lepton asymmetry emerges.

With the basic mechanism outlined, its quantitative description should address the following key questions:

- Concerning $C P$-violation from mixing, the Higgs doublets assume the role of the singlet neutrinos in conventional Leptogenesis. Gauge interactions keep these doublets close to thermal equilibrium. The deviation from equilibrium is mediated by loops involving the singlet neutrinos. Does this induce a $C P$-violating bias in the production of leptons? To answer this, an analysis with accurate account for real intermediate states must be performed. For this purpose, we use the Closed-TimePath (CTP) formalism. The lepton production rate follows from the imaginary part of the diagram in Figure 1, and the correct counting of real and virtual states is incorporated automatically [7 17$]$.

- When the masses of the extra doublets $\phi$ are of Electroweak or TeV scale and $N$ is much heavier, the relevant cut that corresponds to the process $\phi \rightarrow \bar{\ell}+N$ is kinematically forbidden in the vacuum. In a thermal background however, it receives contributions from the distribution functions of $N$ and $\ell$. Again, this can be reliably calculated using CTP methods.

- The resonance for almost degenerate Higgs-doublets should be limited by their widths. We show that the relevant contributions arise from Higgs-flavor violating Yukawa and scalar self-interactions, while the universal flavor conserving gauge interactions do not limit the resonant enhancement. Note that a partial cancellation of the widths that limit the enhancement is familiar from conventional Resonant Leptogenesis [18 21].

\section{The Model and the Mechanism}

The present proposal is realized by the Lagrangian

$$
\begin{aligned}
\mathcal{L} & =\frac{1}{2} \bar{\psi}_{N}\left(\mathrm{i} \not \partial-M_{N}\right) \psi_{N}+\bar{\psi}_{\ell} \mathrm{i} \not \partial \psi_{\ell}+\sum_{k}\left(\partial_{\mu} \phi_{k}\right)\left(\partial^{\mu} \phi_{k}\right) \\
& -\sum_{k l} M_{\phi k l}^{2} \phi_{k}^{*} \phi_{l}-\sum_{m k}\left(Y_{m k} \bar{\psi}_{N} \phi_{k} P_{\mathrm{L}} \psi_{\ell m}+\text { h.c. }\right) .
\end{aligned}
$$




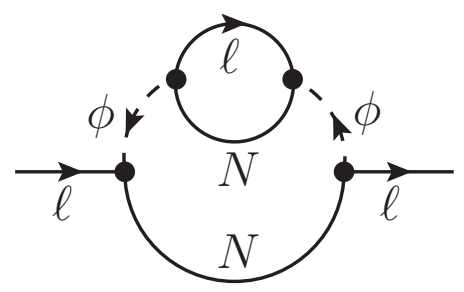

Figure 1: The imaginary part of this Feynman diagram yields the $C P$-violating production rate of lepton flavors.

The spinor $\psi_{N}$ represents the singlet Majorana neutrino $N, \psi_{\ell m}$ with $m=1,2$ two flavors of SM lepton doublets $\ell$ and $\phi_{k}$ with $k=1,2$ are two extra Higgs doublets, besides the one of the SM. The matrix $M_{\phi}^{2}$ is diagonal, what can be achieved by field redefinitions, and indices of the $\mathrm{SU}(2)$ gauge group are suppressed. We take $M_{\phi}^{2}$ as an effective mass, that readily includes thermal corrections.

Using the CTP approach, Leptogenesis can be described by the simple network of equations

$$
\frac{d q_{\ell i}}{d \eta}=S_{\ell i}+W_{\ell_{i}} q_{\ell i}, \quad \frac{d f_{N}(\mathbf{k})}{d \eta}=\mathcal{C}_{N}(\mathbf{k})
$$

Here, $q_{\ell i}$ is the comoving charge density of leptons of flavor $i$ and $f_{X}$ is the comoving distribution function for the particle $X, f_{X}^{\text {eq }}$ are equilibrium distributions and $\eta$ is the conformal time. When $T \gg M_{\phi}$, where $T$ is the temperature, the collision term for singlet neutrinos is [13]

$$
\begin{aligned}
\mathcal{C}_{N}(\mathbf{k}) & =\frac{\sum_{m k}\left|Y_{m k}\right|^{2}}{\sqrt{\mathbf{k}^{2}+M_{N}^{2}}} \int \frac{d^{3} p d^{3} q}{(2 \pi)^{2} 4|\mathbf{p}||\mathbf{q}|} \delta^{4}(k-p-q) 2 k \cdot p \\
& \times\left[1-f_{\ell_{m}}^{\mathrm{eq}}(\mathbf{p})+f_{\phi_{k k}}^{\mathrm{eq}}(\mathbf{q})\right] \times\left[f_{N}^{\mathrm{eq}}(\mathbf{k})-f_{N}(\mathbf{k})\right],
\end{aligned}
$$

and the washout term for the leptons [13]

$$
W_{\ell i}=-\sum_{k}\left|Y_{i k}\right|^{2} \int \frac{d^{3} k d^{3} p d^{3} q 2 p \cdot k}{(2 \pi)^{5} 8|\mathbf{k}| \sqrt{\mathbf{p}^{2}+M_{N}^{2}}|\mathbf{q}|} \delta^{4}(p-k-q)\left[f_{\phi_{k k}}^{\mathrm{eq}}(\mathbf{q})+f_{N}(\mathbf{p})\right] \frac{12 T^{-3} \mathrm{e}^{|\mathbf{k}| / T}}{\left(\mathrm{e}^{|\mathbf{k}| / T}+1\right)^{2}} .
$$

Within the phase-space integrals, we have approximated here $\ell$ and $\phi$ as massless, such that we may substitute $f_{\ell_{1}}^{\mathrm{eq}}=f_{\ell_{2}}^{\mathrm{eq}} \equiv f_{\ell}^{\mathrm{eq}}$ and $f_{\phi_{11}}^{\mathrm{eq}}=f_{\phi_{22}}^{\mathrm{eq}} \equiv f_{\phi}^{\mathrm{eq}}$ by the Fermi-Dirac and Bose-Einstein distributions for massless particles. Of special interest is, of course, the $C P$-violating contribution to the lepton collision term

$$
\begin{aligned}
& \mathcal{C}_{\ell i}^{\mathrm{CPV}}(\mathbf{k})=\frac{\operatorname{Im}\left[Y_{i 1} Y_{j 1}^{*} Y_{j 2} Y_{i 2}^{*}\right]}{M_{\phi 11}^{2}-M_{\phi 22}^{2}} \int \frac{d k^{0} d^{4} p d^{4} q}{(2 \pi)^{9}} \\
& \times\left[\mathrm{i} S_{N}^{>}(p+k) \mathrm{i} S_{\ell i}^{<}(k)-<\leftrightarrow>\right]\left[\mathrm{i} \Delta_{\phi_{11}}^{<}(p) P_{\mathrm{L}} S_{\ell j}^{<}(-p-q) \mathrm{i} S_{N}^{<}(q)-<\leftrightarrow>\right]+1 \leftrightarrow 2,
\end{aligned}
$$


where $i \neq j$. This term can be derived using the methods of Ref. [13], and it enters in Eqs. (2) as $S_{\ell i}=\int d^{3} k \mathcal{C}_{\ell i}^{\mathrm{CPV}} /(2 \pi)^{3}$. Note that $\mathcal{C}_{\ell 1}^{\mathrm{CPV}}=-\mathcal{C}_{\ell 2}^{\mathrm{CPV}}$, as a consequence of lepton number conservation in the diagram of Figure 1. Eventually, the total asymmetry arises from different washout of the two flavors [6, 22 24]. The fermionic and scalar Wightman functions $S_{X}^{<,>}$and $\Delta_{\phi}^{<,>}$describe the phase-space distributions of the various quasiparticles and are given e.g. in Ref. [13]. For the present discussion, we note that in thermal equilibrium, the Kubo-Martin-Schwinger (KMS) relations $S_{X}^{>}(p)=-\mathrm{e}^{p^{0} / T} S_{X}^{<}(p)$ and $\Delta_{\phi}^{>}(p)=\mathrm{e}^{p^{0} / T} \Delta_{\phi}^{<}(p)$ hold, which imply that the last factor in Eq. (5) vanishes in equilibrium. However, when $N$ deviates from equilibrium, this term is proportional to $\delta f_{N}=f_{N}-f_{N}^{\text {eq }}$

The CP-violating source, Eq. (5), corresponds to the insertion of a single loop in the Higgs propagator in Figure 1, The question of how finite width limits the resonance can be answered when resumming these insertions as well as those from the additional interactions. When we denote the resummed propagator of the mixing Higgs fields by $D_{\phi}$, the source term becomes

$$
S_{\ell i}=\sum_{\substack{k, l \\ k \neq l}} Y_{i k}^{*} Y_{i l} \int \frac{d^{4} k d^{4} p d^{4} q \delta^{4}(k+p-q)}{(2 \pi)^{8}} \operatorname{tr}\left[P_{\mathrm{R}} \mathrm{i} S_{N}^{>}(q) \mathrm{i} S_{\ell i}^{<}(k)-<\leftrightarrow>\right] \mathrm{i} D_{\phi k l}(p),
$$

where $i \neq j$. We drop the superscripts $<,>$ on $D$, since we consider here only its offdiagonal components, for which $D^{<} \equiv D^{>}$at the leading, non-vanishing order. Moreover, $\mathrm{i} D_{\phi_{21}}=\left(\mathrm{i} D_{\phi_{12}}\right)^{*}$. The off-diagonal components of the resummed propagator can be obtained from the kinetic equations [20, 21],

$$
\begin{aligned}
& 2 k^{0} \partial_{\eta} \mathrm{i} D_{\phi 12}+\mathrm{i}\left(M_{\phi 11}^{2}-M_{\phi 22}^{2}\right) \mathrm{i} D_{\phi 12} \\
= & -\frac{1}{2} \mathrm{i}\left(\Pi_{\phi 12}^{\dagger 1>}+\Pi_{\phi 12}^{Y>}+\Pi_{\phi 12}^{g>}\right) \mathrm{i}\left(\Delta_{\phi_{11}}^{<}+\Delta_{\phi_{22}}^{<}\right)-\frac{1}{2} \sum_{k} \mathrm{i}\left(\Pi_{\phi_{k k}}^{\sharp>}+\Pi_{\phi_{k k}}^{Y>}+\Pi_{\phi_{k k}}^{g>}\right) \mathrm{i} D_{\phi 12}-<\leftrightarrow>,
\end{aligned}
$$

as a perturbation to the diagonal Wightman functions $\Delta_{\phi_{i i}}^{<,>}$, that are of equilibrium form. The Higgs-flavor violating contributions to the self-energy, that are mediated by additional Yukawa couplings $y$ and scalar self-interactions $\lambda$ are given by $\Pi_{\phi}^{\not 1<,>}$, the contributions mediated by the couplings $Y$ by $\Pi_{\phi}^{Y<,>}$ and those from gauge couplings $g$ by $\Pi_{\phi}^{g<,>}$. The latter are flavor conserving in the sense that when integrating Eq. (7) over $d^{4} k$, the terms involving $\Pi_{\phi}^{g<,>}$ vanish. This is shown in Ref. [14] for flavor-coherent fermions, but the argument can be directly applied to the present scalar case. Unfortunately, this argument does not solve Eq. (17) in general. However, when the lifetime of the virtual Higgs $1 /\left|M_{\phi 11}-M_{\phi 22}\right|$ is much larger than $g T$, the time-scale of kinetic equilibration, the off-diagonal components of $D$ follow a kinetic equilibrium distribution [14],

$$
\mathrm{i} D_{\phi 12}(p)=2 \pi \delta\left(p^{2}-\bar{M}_{\phi}^{2}\right) \frac{\mu_{\phi 12}}{T} \frac{\operatorname{sign}\left(p^{0}\right) \mathrm{e}^{\left|p^{0}\right| / T}}{\left(\mathrm{e}^{\left|p^{0}\right| / T}-1\right)^{2}},
$$


where $\bar{M}_{\phi}=\left|M_{\phi 11}-M_{\phi 22}\right| / 2$ and for $M_{\phi} \ll T$,

$$
q_{\phi 12}=\frac{\mu_{\phi 12} T^{2}}{3}=2 \int \frac{d^{4} k}{(2 \pi)^{4}} k^{0} \mathrm{i} D_{\phi_{12}}(k) .
$$

Note that in kinetic equilibrium, the terms in Eq. (7) that involve $\Pi_{\phi}^{g<,>}$ readily cancel before integration over $d^{4} k$. From Eq. (7), when neglecting the derivative with respect to $\eta$, it then follows

$$
\begin{aligned}
q_{\phi 12} & =\frac{4 \mathrm{i} \sum_{j} Y_{j 1} Y_{j 2}^{*}}{M_{\phi 11}^{2}-M_{\phi 22}^{2}+\mathrm{i} T \bar{\Gamma}^{\natural 1}} \int \frac{d^{3} k d^{3} p d^{3} q \delta^{4}(k+p-q)}{(2 \pi)^{5} 8|k||p| \sqrt{|\mathbf{q}|^{2}+M_{N}^{2}}} \\
& \times k^{0} 2 p \cdot q\left[1-f_{\ell}^{\mathrm{eq}}(\mathbf{p})+f_{\phi}^{\mathrm{eq}}(\mathbf{k})\right] \delta f_{N}(\mathbf{q}),
\end{aligned}
$$

where $\bar{\Gamma}^{\natural}$ is a weighted average of $\Pi_{\phi}^{\sharp 1<,>}$ and $\Pi_{\phi}^{Y<,>}$, which we estimate below. This limits the resonant enhancement of the asymmetry. The important point is that the flavor-conserving gauge interactions do not contribute to $\bar{\Gamma}^{\natural 1}$ at leading order.

\section{Lepton Asymmetry}

Though to this end, the discussion has been more general, we now restrict to a phenomenologically interesting region of parameter space where analytic approximations of good accuracy are available. In the strong washout regime, the right handed neutrino is non-relativistic, i.e. $M_{N} \gg T$, when the asymmetry freezes out. Moreover, we assume that $M_{\phi i i} \ll M_{N}$. Then, we can neglect the masses of the Higgs particles in the phase space integrals and only keep them in the resonant enhancement factors. As $M_{N} \gg T$, the decay products are of energy much larger than $T$ as well. Therefore, we may replace the quantum-statistical distribution functions by Maxwell distributions.

Moreover, we describe the deviation of the right-handed neutrino from equilibrium by a pseudo-chemical potential, $\delta f_{N}(\mathbf{k}) \approx \exp \left(\sqrt{\mathbf{k}^{2}+M_{N}^{2}}\right) \mu_{N} / T$. Even though there are no interactions that drive $N$ toward kinetic equilibrium, this empirically proves to be a good approximation [13, 25]. We then obtain the off-diagonal charges in the mixing Higgs-system from Eq. (10),

$$
q_{\phi 12}=\frac{\mathrm{i} \sum_{j} Y_{j 1} Y_{j 2}^{*}}{M_{\phi 11}^{2}-M_{\phi 22}^{2}} \frac{\mu_{N} M_{N}^{7 / 2} T^{1 / 2}}{16 \sqrt{2} \pi^{5 / 2}} \mathrm{e}^{-M_{N} / T},
$$

where we approximate $\bar{\Gamma}^{\boxplus 1} \ll\left|M_{\phi 11}-M_{\phi 22}\right|$, which turns out as generic. Substitution into Eqs. (819) and (6) yields

$$
S_{\ell i}=\frac{3 \mu_{N} M_{N}^{6}}{512 \pi^{5} T} \frac{\operatorname{Im}\left[Y_{i 1} Y_{j 1}^{*} Y_{j 2} Y_{i 2}^{*}\right]}{M_{\phi 11}^{2}-M_{\phi 22}^{2}} \mathrm{e}^{-2 M_{N} / T} .
$$


Now we are set to follow the standard routine for calculating the asymmetry in the strong washout regime [26, 27]. The expanding background is taken account of following Ref. [13], and Eqs. (21) simplify to

$$
\begin{aligned}
\frac{d Y_{\ell i}}{d z} & =\bar{S}_{\ell i}\left(Y_{N}-Y_{N}^{\mathrm{eq}}\right)+\bar{W}_{\ell i} Y_{\ell i} \\
\frac{d Y_{N}}{d z} & =\overline{\mathcal{C}}_{N}\left(Y_{N}-Y_{N}^{\mathrm{eq}}\right)
\end{aligned}
$$

where $Y_{\ell i}=4 q_{\ell i} / s, Y_{N}=2 \int d^{3} k f_{N}(\mathbf{k}) /\left((2 \pi)^{3} s\right.$ ) (the factors account for spin and $\mathrm{SU}(2)$ degrees of freedom), $z=m_{N} / T$ and $s$ is the entropy density. The integrated distributions and collision terms that occur here can be obtained from Eqs. (3,4,12) and are explicitly given by

$$
\begin{aligned}
Y_{N}^{\mathrm{eq}} & =2^{-1 / 2} \pi^{-3 / 2} a_{\mathrm{R}}^{3} z^{3 / 2} \mathrm{e}^{-z} / s, \\
\overline{\mathcal{C}} & =-\frac{1}{8 \pi} \operatorname{tr}\left[Y Y^{\dagger}\right] \frac{a_{\mathrm{R}}}{M_{N}} z, \\
\bar{W}_{\ell i} & =3 \times 2^{-9 / 2} \pi^{-5 / 2} \sum_{k} Y_{i k} Y_{k i}^{\dagger} \frac{a_{\mathrm{R}}}{M_{N}} z^{5 / 2} \mathrm{e}^{-z}, \\
\bar{S}_{i} & =\frac{3}{2^{13 / 2} \pi^{7 / 2}} \frac{\operatorname{Im}\left[Y_{i 1} Y_{j 1}^{*} Y_{j 2} Y_{i 2}^{*}\right]}{M_{\phi 11}^{2}-M_{\phi 22}^{2}} a_{\mathrm{R}} M_{N} z^{5 / 2} \mathrm{e}^{-z},
\end{aligned}
$$

where $a_{\mathrm{R}}=(1 / 2) m_{\mathrm{Pl}}\left(\pi^{3} g_{\star} / 45\right)^{-1 / 2}, m_{\mathrm{Pl}}=1.22 \times 10^{19} \mathrm{GeV}$ is the Planck mass and $g_{\star}$ the number of relativistic degrees of freedom.

The Eqs. (13) can be formally integrated and then evaluated employing Laplace's steepest descent method with the result

$$
\begin{aligned}
Y_{\ell i}(z=\infty) & =\frac{\operatorname{Im}\left[Y_{i 1} Y_{j 1}^{*} Y_{j 2} Y_{i 2}^{*}\right]}{\operatorname{tr}\left[\mathrm{YY}^{\dagger}\right]} \frac{135 M_{N}^{2}}{64 \pi^{6} g_{\star}} \frac{1}{M_{\phi 11}^{2}-M_{\phi 22}^{2}} \sqrt{z_{\mathrm{f} i}^{-1 / 2} \mathrm{e}^{z_{\mathrm{f} i}}} \mathrm{e}^{-2 z_{\mathrm{f} i}-\int_{\mathrm{f}_{\mathrm{f} i}}^{\infty} d z B_{i} z^{5 / 2} \mathrm{e}^{-z}} \\
B_{i} & =\sum_{k} Y_{i k} Y_{i k}^{*} \frac{9}{32 \pi^{4}} \sqrt{\frac{5}{2 g_{\star}}} \frac{m_{\mathrm{Pl}}}{M_{N}} \\
z_{\mathrm{f} i} & =-\frac{5}{2} W_{-1}\left((-2 / 5) \times\left(2 / B_{i}\right)^{2 / 5}\right)
\end{aligned}
$$

where $W_{-1}$ is the lower branch of the product logarithm. It should be clear that asymmetries $Y_{\ell i}$ of order $10^{-10}$ or larger can easily be obtained, $c f$. Section 4 for more details. The temperature at freeze-out is given by $M_{N} / z_{\mathrm{f} i}$, which is sufficiently accurately approximated when $B_{i} \gtrsim 1$, corresponding to the strong washout regime. In the exponent of Eq. (15a), there is the term $-2 z_{\mathrm{f} i}$ rather than $-z_{\mathrm{f} i}$ for conventional Leptogenesis [26, 27], because the cut that leads to the asymmetry is kinematically forbidden in the vacuum, 
what gives an additional Maxwell suppression. It is crucial that $B_{1} \neq B_{2}$, such that a cancellation of the flavored asymmetries is avoided. (See however Ref. [28] for a loophole.)

Before applying the formula (15a), we must consider the mass difference and the flavor-violating width, that limit the resonant enhancement. For $M_{\phi} \ll T$, the relevant contributions to the asymptotic (momenta much larger than $T$ ) Higgs mass are

$$
M_{\phi i i}^{2}=m_{\phi i i}^{2}+\left(\sum_{k} \frac{\lambda_{k i}}{2}+\sum_{j} \frac{y_{j i}^{2}}{12}+\frac{3 g_{\mathrm{L}}^{2}}{16}+\frac{g_{Y}^{2}}{16}\right) T^{2}
$$

where $\lambda_{k i}$ denotes the coupling to the Higgs doublet $k, y_{j i}$ the coupling to the pair of chiral fermions $i, m_{\phi i i}^{2}$ is the vacuum mass and $g_{\mathrm{L}}, g_{Y}$ are the $\mathrm{SU}(2)$ and $\mathrm{U}(1)$ gauge couplings of the SM. Assuming that $g_{\mathrm{L}}^{2}, g_{Y}^{2} \gg Y^{2}, y^{2}, \lambda$, the leading contributions to the flavor-violating width are $\bar{\Gamma}^{1}=\mathcal{O}\left(g_{\mathrm{L}}^{2}, g_{Y}^{2} \times\left(y^{2}, Y^{2}, \lambda\right)\right) \times T$. This is because the damping of the flavor correlations of the Higgs fields is kinematically suppressed by the small zerotemperature and thermal masses (compared to $T$ ) in $1 \leftrightarrow 2$ processes or by additional powers of couplings in $2 \leftrightarrow 2$ scattering processes. A full calculation of $\Gamma^{\sharp 1}$ is presently still very challenging, $c f$. the discussion in Ref. [29]. In conclusion, the suppression of the resonance through thermal masses is generically stronger than the effect of Higgs flavorviolating damping rates, when barring accidental cancellations. In case $M_{\phi}$ is dominated by the zero-temperature mass $m_{\phi}$, the resonance is therefore implied by the hierarchy between $M_{N}$ and $m_{\phi}$, whereas otherwise, it is implied by the small Higgs-flavor violating couplings $y$ and $\lambda$.

\section{Resonant Enhancement Required for the Observed Asymmetry}

The observed value for the baryon asymmetry of the Universe is [30]

$$
\eta_{\mathrm{B}}=\frac{n_{\mathrm{B}}}{n_{\gamma}}=(6.16 \pm 0.15) \times 10^{-10},
$$

where $n_{\mathrm{B}}$ is the baryon number density and $n_{\gamma}$ the number of photons in the cosmic microwave background. How small does $\left|M_{\phi 11}^{2}-M_{\phi 22}^{2}\right|$ have to be in order to account for the observed asymmetry for a given singlet neutrino mass $M_{N}$ ? Leptogenesis for the flavor $i$ is typically most efficient in the regime between strong and weak washout, and this transition occurs close to the point where the product logarithm $-W_{-1}$ takes its smallest real values [27], i.e. when

$$
\frac{2}{5}\left(\frac{2}{B_{i}}\right)^{\frac{2}{5}}=\frac{1}{\mathrm{e}} \Rightarrow z_{\mathrm{f} i}=\frac{5}{2}
$$


Moreover, the $C P$ asymmetry is maximized when $\left|Y_{\ell 1}\right|=\left|Y_{\ell 2}\right|$ and the $C P$-violating phase is maximal. The value $z_{\mathrm{f} i}=\frac{5}{2}$ is then obtained for

$$
\left|Y_{i 1}\right|^{2}=\left|Y_{i 2}\right|^{2}=\frac{32 \pi^{4}}{9} \mathrm{e}^{\frac{5}{2}} \sqrt{2 g_{\star} / 5} \frac{M_{N}}{m_{\mathrm{Pl}}}
$$

Up to the numerical factor, this relation is identical to the corresponding one that determines the transiton between strong and weak washout regimes for conventional Leptogenesis. Just as in the conventional scenario, it therefore implies that the couplings $Y_{\ell i}$ are sufficiently small, such that they do not jeopardize the upper bounds on the active neutrino masses, even when the extra Higgs bosons acquire sizeable vacuum expectation values after Electroweak Symmetry Breaking. Besides, we assume that the couplings of the lepton flavor $j$ are much larger than for the flavor $i$, i.e. $\left|Y_{j 1,2}\right| \gg\left|Y_{i 1,2}\right|$, such that the flavor $j$ suffers a stronger washout and its contribution to the final asymmetry may be neglected. Under these assumptions, the first factor in Eq. (15a) is independent of the magnitude of $Y_{j 1,2}$, because $\left|Y_{j 1}^{*} Y_{j 2}\right| / \operatorname{tr}\left[Y Y^{\dagger}\right] \approx \frac{1}{2}$.

Putting these assumptions and approximations together, we evaluate Eq. (15a) with the result

$$
Y_{\ell i}=9.8 \times 10^{-4} \frac{M_{N}^{3}}{\sqrt{g_{\star}} m_{\mathrm{Pl}}\left(M_{\phi 11}^{2}-M_{\phi 22}^{2}\right)}=9.2 \times 10^{-5} \frac{M_{N}^{3}}{m_{\mathrm{Pl}}\left(M_{\phi 11}^{2}-M_{\phi 22}^{2}\right)},
$$

where we have taken $g_{\star}=114.75$ as the number of relativistic degrees of freedom in the Standard Model with two additional Higgs doublets at high temperatures $\left(N_{\phi}=3\right)$.

Now for definiteness, we assume that the Electroweak phase transition is of strongly first order, such that [28, 31]

$$
\eta_{\mathrm{B}}=7.04 \times Y_{\ell i} \frac{24+4 N_{\phi}}{66+13 N_{\phi}} .
$$

When the sphaleron freeze-out occurs after Electroweak Symmetry Breaking, this conversion factor is slightly different, which should however not be relevant within the accuracy of the present approximations.

From Eqs. (17],19]20), we obtain the following upper bound on the splitting of the effective (i.e. including thermal corrections) Higgs masses

$$
\left|M_{\phi 11}^{2}-M_{\phi 22}^{2}\right|<3.0 \times 10^{-14} \mathrm{GeV}^{2} \frac{M_{N}^{3}}{(1 \mathrm{GeV})^{3}}
$$

Under the assumption that the zero-temperature masses are small compared to the effective masses, it is also illustrative to use Eq. (16) to turn this into a bound on the coupling constants

$$
\frac{1}{2}\left|\sum_{k}\left(\lambda_{k i}^{2}-\lambda_{k j}^{2}\right)\right|+\frac{1}{12}\left|\sum_{l}\left(y_{l i}^{2}-y_{l j}^{2}\right)\right|<2.3 \times 10^{6} \frac{M_{N}}{m_{\mathrm{Pl}}}
$$


It should be kept in mind that the scenario relies on flavor effects, i.e. that there exist two effectively non-degenerate lepton flavors at the time of Leptogenesis. Therefore, $M_{N}$ should be of order $10^{12} \mathrm{GeV}$ or smaller, such that at least the $\tau$-Yukawa coupling is in equilibrium. (However, due to the extended Higgs sector, it is also conceivable that the flavor-degeneracy may be already broken at higher temperatures.) It is interesting to notice that when $M_{N}$ is of order $10^{12} \mathrm{GeV}$, no strong suppression of the Higgs flavorviolating rates is required in order to explain the observed asymmetry. We note that of course, in the absence of a symmetry that suppresses flavor-changing neutral currents, there are restrictive upper bounds on these Yukawa couplings or lower bounds on the zero-temperature masses, $c f$. Ref. [32] for a recent review.

The results of this Section illustrate that the resonant enhancement of $C P$-violation in the present mechanism is of a quite different origin than in conventional scenarios of Resonant Leptogenesis. While in the latter case, a small mass difference of at least two singlet neutrinos must be imposed by virtue of tuning or a symmetry, in the present scenario, small zero-temperature Higgs masses compared to the singlet neutrino mass and small Higgs flavor violating couplings are required. The tuning responsible for the smallness of the zero-temperature Higgs masses can be identified with the tuning that is required in order to stabilize the Electroweak scale compared to higher energy scales. The suppression of the Higgs-flavor violating couplings of the additional doublets is not well-motivated, unless one appeals to new symmetries, but it is at the same time required by the suppression of flavor changing neutral currents [32].

\section{$5 \quad$ Summary and Discussion}

Multiple Higgs doublets generically contribute resonantly enhanced $C P$ violation to flavored Leptogenesis. The resonance is typically controlled by the the effective thermal mass degeneracy, that can be inferred from Eq. (16), which is to be substituted into the expression for the final asymmetry (15a) in the strong washout regime.

The intuitive picture of the present mechanism is that an out-of-equilibrium distribution $\delta f_{N}$ induces off-diagonal correlations of the Higgs flavors. Because gauge interactions are flavor blind, these do not directly damp these correlations, but only force them into kinetic equilibrium. Note that a partial cancellation of widths is also familiar from the decay asymmetry in conventional Resonant Leptogenesis [18 21]. The decay of the flavor-coherent Higgs particles leads to a lepton flavor asymmetry.

While we calculate the asymmetry for $M_{N} \gg T$, we note that the present model becomes similar to the one underlying Dirac Leptogenesis [33] when $M_{N} \rightarrow 0$. In Ref. [33] however, the question of resonant enhancement is not addressed, while Ref. 34] misses that the resonance is spoilt by large Yukawa couplings. In both, Refs. [33, 34], it is proposed that instead of $N$, a right-handed electron propagates in the loop, which would be in thermal equilibrium, such that no asymmetry in $\ell$ can arise according to the discussion of Eq. (5) and KMS.

The present work builds on two key aspects of more or less recently achieved progress 
in Leptogenesis: the observation of the importance of flavor [6, 22 24] and the reliable treatment of leading statistical corrections [7-17], that are crucial for the source terms (12) and (14d).

While extra Higgs doublets may be experimentally observable, the singlet neutrino generically is yet very heavy and too weakly coupled in order to be discovered. It would therefore be interesting to conceive of models that connect the present mechanism to the phenomenology of neutrino oscillations, in order to obtain further constraints. We close with two more speculative remarks: First, close-to degenerate states other than Higgs doublets, that may be produced experimentally via gauge interactions, might be connected with baryogenesis. Since their connection to the lepton sector must be either indirect or mediated by non-renormalizable operators, the analysis of such models is presumably less straightforward than in the present case. Second, it would be interesting to re-consider Electroweak Baryogenesis from mixing particles, where it is often assumed that the resonance is limited by gauge interactions, which is not the case for the present scenario of Leptogenesis.

\section{Acknowledgments}

The author is grateful to Martin Beneke and Marco Drewes for valuable comments on the manuscript and acknowledges support by the Gottfried Wilhelm Leibniz programme of the Deutsche Forschungsgemeinschaft.

\section{References}

[1] L. Covi, E. Roulet and F. Vissani, "CP violating decays in leptogenesis scenarios," Phys. Lett. B 384 (1996) 169 [hep-ph/9605319].

[2] M. Flanz, E. A. Paschos, U. Sarkar and J. Weiss, "Baryogenesis through mixing of heavy Majorana neutrinos," Phys. Lett. B 389 (1996) 693 hep-ph/9607310].

[3] A. Pilaftsis, "Resonant CP violation induced by particle mixing in transition amplitudes," Nucl. Phys. B 504 (1997) 61 hep-ph/9702393.

[4] A. Pilaftsis, "CP violation and baryogenesis due to heavy Majorana neutrinos," Phys. Rev. D 56 (1997) 5431 hep-ph/9707235.

[5] A. Pilaftsis and T. E. J. Underwood, "Resonant leptogenesis," Nucl. Phys. B 692 (2004) 303 [hep-ph/0309342].

[6] A. Pilaftsis and T. E. J. Underwood, "Electroweak-scale resonant leptogenesis," Phys. Rev. D 72 (2005) 113001 hep-ph/0506107.

[7] W. Buchmuller and S. Fredenhagen, "Quantum mechanics of baryogenesis," Phys. Lett. B 483, 217 (2000) hep-ph/0004145. 
[8] A. De Simone and A. Riotto, "Quantum Boltzmann Equations and Leptogenesis," JCAP 0708 (2007) 002 [hep-ph/0703175].

[9] M. Garny, A. Hohenegger, A. Kartavtsev and M. Lindner, "Systematic approach to leptogenesis in nonequilibrium QFT: vertex contribution to the CP-violating parameter," Phys. Rev. D 80 (2009) 125027 [arXiv:0909.1559 [hep-ph]].

[10] M. Garny, A. Hohenegger, A. Kartavtsev and M. Lindner, "Systematic approach to leptogenesis in nonequilibrium QFT: self-energy contribution to the CP-violating parameter," Phys. Rev. D 81 (2010) 085027 [arXiv:0911.4122 [hep-ph]].

[11] A. Anisimov, W. Buchmüller, M. Drewes and S. Mendizabal, "Leptogenesis from Quantum Interference in a Thermal Bath," Phys. Rev. Lett. 104 (2010) 121102 arXiv:1001.3856 [hep-ph]].

[12] M. Garny, A. Hohenegger, A. Kartavtsev, "Medium corrections to the CP-violating parameter in leptogenesis," Phys. Rev. D81 (2010) 085028. arXiv:1002.0331 [hep$\mathrm{ph}]]$.

[13] M. Beneke, B. Garbrecht, M. Herranen and P. Schwaller, "Finite Number Density Corrections to Leptogenesis," Nucl. Phys. B 838 (2010) 1 arXiv:1002.1326 [hep$\mathrm{ph}]]$.

[14] M. Beneke, B. Garbrecht, C. Fidler, M. Herranen and P. Schwaller, "Flavoured Leptogenesis in the CTP Formalism," Nucl. Phys. B 843 (2011) 177 arXiv:1007.4783 [hep-ph]].

[15] M. Garny, A. Hohenegger and A. Kartavtsev, "Quantum corrections to leptogenesis from the gradient expansion," arXiv:1005.5385 [hep-ph].

[16] B. Garbrecht, "Leptogenesis: The Other Cuts," Nucl. Phys. B847 (2011) 350-366. arXiv:1011.3122 [hep-ph]].

[17] A. Anisimov, W. Buchmuller, M. Drewes and S. Mendizabal, "Quantum Leptogenesis I," Annals Phys. 326 (2011) 1998 arXiv:1012.5821 [hep-ph]].

[18] W. Buchmuller and M. Plumacher, "CP asymmetry in Majorana neutrino decays," Phys. Lett. B 431 (1998) 354 [hep-ph/9710460].

[19] A. Anisimov, A. Broncano and M. Plumacher, "The CP-asymmetry in resonant leptogenesis," Nucl. Phys. B 737 (2006) 176 [hep-ph/0511248].

[20] B. Garbrecht and M. Herranen, "Effective Theory of Resonant Leptogenesis in the Closed-Time-Path Approach," Nucl. Phys. B 861 (2012), 17. arXiv:1112.5954[hep$\mathrm{ph}]]$.

[21] M. Garny, A. Kartavtsev and A. Hohenegger, "Leptogenesis from first principles in the resonant regime," arXiv:1112.6428 [hep-ph]. 
[22] T. Endoh, T. Morozumi and Z. -h. Xiong, "Primordial lepton family asymmetries in seesaw model," Prog. Theor. Phys. 111 (2004) 123 [hep-ph/0308276].

[23] A. Abada, S. Davidson, F. X. Josse-Michaux, M. Losada and A. Riotto, "Flavour Issues in Leptogenesis," JCAP 0604, 004 (2006) arXiv:hep-ph/0601083.

[24] E. Nardi, Y. Nir, E. Roulet and J. Racker, "The importance of flavor in leptogenesis," JHEP 0601, 164 (2006) arXiv:hep-ph/0601084.

[25] A. Basboll and S. Hannestad, "Decay of heavy Majorana neutrinos using the full Boltzmann equation including its implications for leptogenesis," JCAP 0701 (2007) 003 [hep-ph/0609025.

[26] E. W. Kolb and M. S. Turner, "Grand Unified Theories and the Origin of the Baryon Asymmetry," Ann. Rev. Nucl. Part. Sci. 33 (1983) 645.

[27] W. Buchmuller, P. Di Bari and M. Plumacher, "Leptogenesis for pedestrians," Annals Phys. 315 (2005) 305 hep-ph/0401240.

[28] D. J. H. Chung, B. Garbrecht and S. Tulin, "The Effect of the Sparticle Mass Spectrum on the Conversion of B-L to B," JCAP 0903 (2009) 008 arXiv:0807.2283 [hep-ph]].

[29] A. Anisimov, D. Besak and D. Bodeker, "Thermal production of relativistic Majorana neutrinos: Strong enhancement by multiple soft scattering," JCAP 1103 (2011) 042 [arXiv:1012.3784 [hep-ph]].

[30] E. Komatsu et al. [WMAP Collaboration], "Seven-Year Wilkinson Microwave Anisotropy Probe (WMAP) Observations: Cosmological Interpretation," Astrophys. J. Suppl. 192 (2011) 18 [arXiv:1001.4538 [astro-ph.CO]].

[31] J. A. Harvey and M. S. Turner, "Cosmological baryon and lepton number in the presence of electroweak fermion number violation," Phys. Rev. D 42 (1990) 3344.

[32] G. C. Branco, P. M. Ferreira, L. Lavoura, M. N. Rebelo, M. Sher and J. P. Silva, "Theory and phenomenology of two-Higgs-doublet models," arXiv:1106.0034 [hep$\mathrm{ph}$.

[33] K. Dick, M. Lindner, M. Ratz and D. Wright, "Leptogenesis with Dirac neutrinos," Phys. Rev. Lett. 84 (2000) 4039 [hep-ph/9907562].

[34] A. Bechinger and G. Seidl, "Resonant Dirac leptogenesis on throats," Phys. Rev. D 81 (2010) 065015 arXiv:0907.4341 [hep-ph]]. 